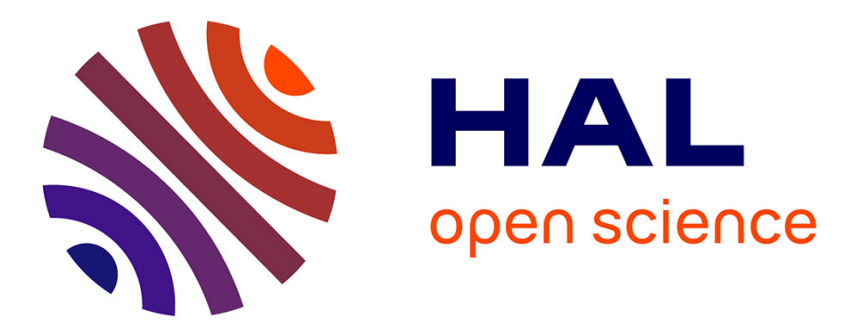

\title{
Preparation of the Three C1-C7, C8-C15, and C16-N22 Fragments of the Hsp90 Inhibitor Herbimycin A
}

Sylvie Centonze-Audureau, François-Hugues Porée, Jean-François Betzer, Jean-Daniel Brion, Ange Pancrazi, Janick Ardisson

\section{To cite this version:}

Sylvie Centonze-Audureau, François-Hugues Porée, Jean-François Betzer, Jean-Daniel Brion, Ange Pancrazi, et al.. Preparation of the Three C1-C7, C8-C15, and C16-N22 Fragments of the Hsp90 Inhibitor Herbimycin A. SYNLETT, 2005, 2005 (06), pp.0981-0985. 10.1055/s-2005-864804 . hal03333714

\section{HAL Id: hal-03333714 https://hal.science/hal-03333714}

Submitted on 3 Sep 2021

HAL is a multi-disciplinary open access archive for the deposit and dissemination of scientific research documents, whether they are published or not. The documents may come from teaching and research institutions in France or abroad, or from public or private research centers.
L'archive ouverte pluridisciplinaire HAL, est destinée au dépôt et à la diffusion de documents scientifiques de niveau recherche, publiés ou non, émanant des établissements d'enseignement et de recherche français ou étrangers, des laboratoires publics ou privés. 
Preparation of the Three C1-C7, C8-C15 and C16-N22 Fragments of the Hsp90 Inhibitor Herbimycin A.

Sylvie Centonze-Audureau, François-Hugues Porée, Jean-François Betzer, Jean-Daniel Brion, ${ }^{*}{ }^{*}$ Ange Pancrazi, Janick Ardisson $b^{*}$

a) CNRS-BIOCIS, Université de Châtenay-Malabry, Faculté de Pharmacie, 92296 Châtenay-Malabry, France

Tel-Fax: (33) 0146835398

b) "Laboratoire de Synthèse Organique Selective et Chimie Organométallique", CNRS-UCP-ESCOM, UMR 8123

ESCOM, Bat E 13 Bd de 1'Hautil, 95092 Cergy-Pontoise, France

Tel-Fax: 0130756186 E-Mail: janick.ardisson@chim.u-cergy.fr

\begin{abstract}
:
The construction of the three C16-N22 2, C1-C7 23 and C8-C15 32 segments of the Hsp90 inhibitor herbimycin A 1 is reported. 1-Iodo-3nitro-2,5-diphenol compound 2 was obtained in 55\% yield for 3 steps from the commercially available diiodo derivative 7. Reaction between 1,1-dibromo-alkene 22 and vinyltin 17a using $\mathrm{Pd}\left(\mathrm{PPh}_{3}\right)_{4}$ or $\mathrm{Pd}\left(\mathrm{CH}_{3} \mathrm{CN}\right)_{2} \mathrm{Cl}_{2} / \mathrm{CuI} /$ diisopropylethylamine, in toluene or DMF at $85^{\circ} \mathrm{C}$, led to enyne 23 in $63 \%$ yield $(19 \%$ overall yield from isopropylidene glyceraldehyde). The synthesis of the $\mathrm{C} 8-\mathrm{C} 15$ sub-unit 32 was performed in 13 steps in $3.4 \%$ overall yield from the commercially available ester $\mathbf{2 4}$, with a Hoppe crotylation as a key step.
\end{abstract}

Molecular chaperones such as heat shock protein 90 (HSP90) assist the folding, maturation and subcellular localisation of their client proteins, and target damaged proteins for degradation via the proteasome. Therefore, the inhibition of Hsp90 provides a novel approach toward regulating crucial enzymes involved in the progression of cancer. The benzoquinoid ansamycins, such as herbimycin A and geldanamycin, have recently been identified as inhibitors of the Hsp90 folding process. Consequently, these molecules are getting a lot of attention in the literature. 1

Herbimycin A 12 was isolated from the fermentation broth of Streptomyces hygroscopicus strain AM-3672, and exhibits pronounced antitumor and antiangiogenic properties. At this time, only two syntheses of herbimycin A 1 has been reported. 3

Our synthetic plan developed for herbimycin A 1 involves the convergent approach depicted in Scheme 1. Our intention was to employ metal-catalysed coupling reactions to form both the $\mathrm{C} 15-\mathrm{C} 16$ 
bond between the Northern C16-N22 aromatic fragment 2 and the Southern C8-C15 side chain 3, and the C7-C8 link between the Eastern C1-C7 dienyl sub-unit 4 and segment 3. A macrolactamization was programmed for the final cyclization step at N22-C1.

The present work describes the preparation of C16-N22 diphenol 2, C1-C7 enyne 6 and C8-C15 alcohol 5 (as precursors of compounds 4 and $\mathbf{3}$, respectively).

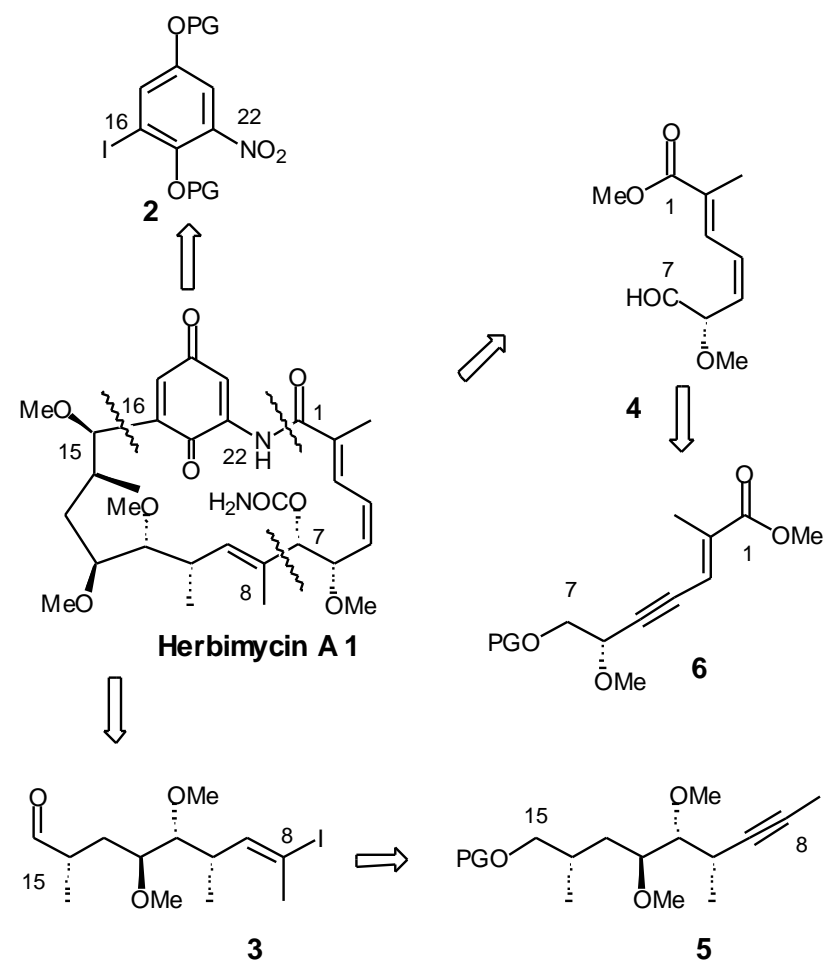

\section{Scheme 1}

Northern fragment 2 preparation was effected in 3 steps in 55\% overall yield from the commercially available diiodo compound 7 . Mono-nitration of 7 under classical conditions led to nitro-aldehyde $\mathbf{8}$ in $80 \%$ yield. In order to generate the diphenol derivative 9, we took advantage of the aldehyde function to perform a Baeyer-Villiger oxidation. This reaction cleanly delivered compound 9 in $80 \%$ yields.

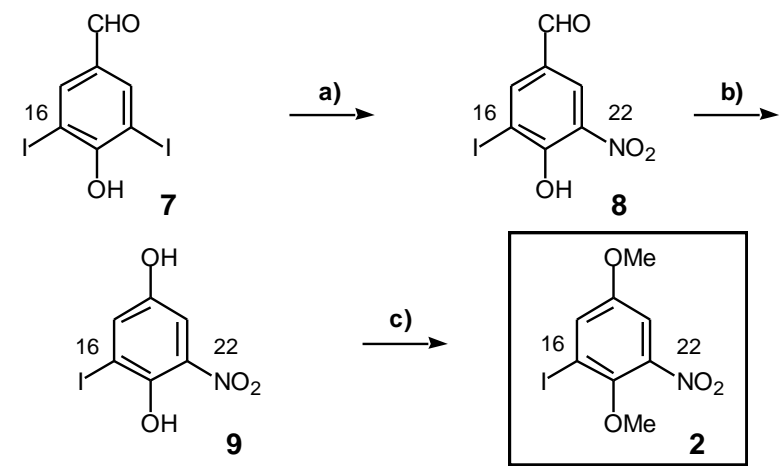

a) $\mathrm{NaNO}_{2}$ ( 1 equiv.), $\mathrm{AcOH} 80^{\circ} \mathrm{C}, 15 \mathrm{~h}, \mathbf{8 0} \%$. b) $m$-CPBA (3 equiv.), $\mathrm{CH}_{2} \mathrm{Cl}_{2} 4^{\circ} \mathrm{C}, 15 \mathrm{~h}, \mathbf{8 0} \%$. c) $\mathrm{KOH}, \mathrm{CH}_{3} \mathrm{I}, \mathrm{DMF}, 20^{\circ} \mathrm{C}, 4 \mathrm{~h}, \mathbf{8 5 \%}$. 


\section{Scheme 2}

The last step for the preparation of C16-N22 Northern fragment 2 was achieved by methylation of 9 with $\mathrm{KOH} / \mathrm{CH}_{3} \mathrm{I}$ in DMF at $20^{\circ} \mathrm{C}$ for 4 $\mathrm{h}$, in $85 \%$ yield. 4

For the Eastern fragment 4 synthesis, a Stille $\operatorname{Pd}(0)$-catalysed coupling reaction was planed between vinyl partners 10 and $\mathbf{1 1}$ (Scheme 3), or between 10 and acetylenic compound 12. In this approach, a partial reduction of the resulting enyne $\mathbf{6}$ is required to reach the desired compound 4.<smiles>COC(=O)c1ccc(O)cc1</smiles>

4<smiles>C=CC(C)OC(C)C#CC=C(C)C(C)OC</smiles>

6
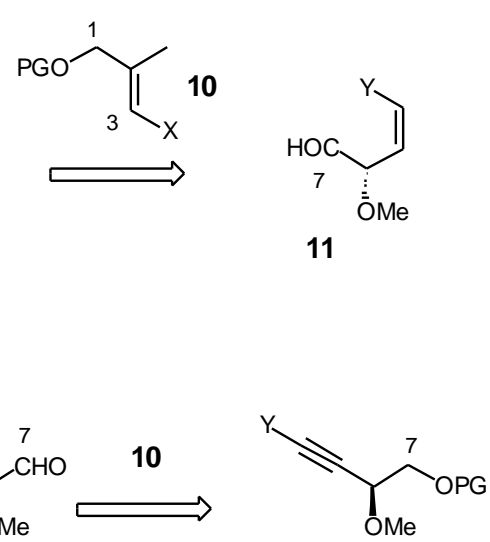

12

\section{Scheme 3}

For synthesis of $\mathbf{1 1}$ and $\mathbf{1 2}$, isopropylidene glyceraldehyde $\mathbf{1 3}^{5}$ was elected as an appropriate starting material to introduce the C-6 centre (Scheme 4). Application of the Corey-Fuchs method6 then led to dibromo alkene 14, which is a versatile intermediate. 7

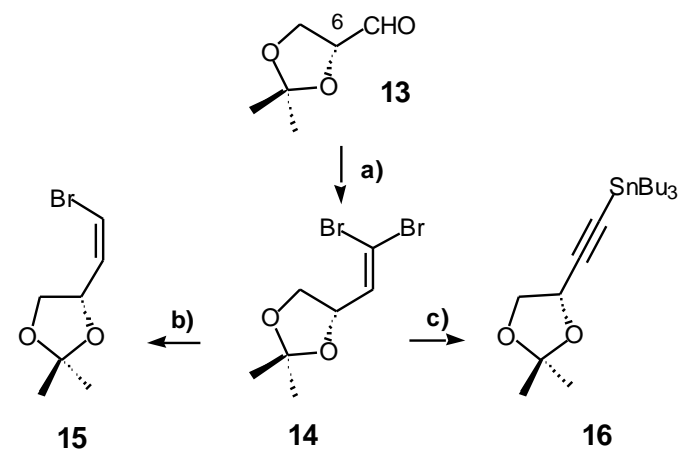

a) $\mathrm{Zn}$ (3 equiv.), $\mathrm{CBr}_{4}$ (3 equiv.), $\mathrm{PPh}_{3}$ (3 equiv.), $\mathrm{CH}_{2} \mathrm{Cl}_{2}, 20^{\circ} \mathrm{C} 12 \mathrm{~h}$, 65\%. b) $\mathrm{Bu}_{3} \mathrm{SnH}$ (1.1 equiv.), $\mathrm{Pd}\left(\mathrm{PPh}_{3}\right)_{4}, \mathrm{THF}, 20^{\circ} \mathrm{C} 20 \mathrm{~min} \mathbf{9 0 \%}$. c) $n$ $\mathrm{BuLi}$ (3 equiv.), $-40^{\circ} \mathrm{C} 2 \mathrm{~h}, \mathrm{Bu}_{3} \mathrm{SnCl}, 20^{\circ} \mathrm{C}, 1 \mathrm{~h}, \mathbf{9 5 \%}$.

\section{Scheme 4}

1,1-Dibromo-1-alkene 14 delivered pure (Z)-vinyl bromide 15 under $\mathrm{Bu}_{3} \mathrm{SnH} / \mathrm{Pd}(0)$ conditions, in $90 \%$ yield (Scheme 5). 8 Besides, acetylenic compounds 167 (95\% yield) was easily obtained by $n$-BuLi treatment of $\mathbf{2 0}$, followed by $\mathrm{Bu}_{3} \mathrm{SnCl}$ trap. 
However, we were disappointed to find out that Stille coupling reaction of vinylbromide $\mathbf{1 5}$ and vinyltin $17 \mathbf{a}^{9}$ did not lead to the expected coupling product 18, even with $\mathrm{Pd}\left(\mathrm{PPh}_{3}\right)_{4}$ or $\mathrm{Pd}\left(\mathrm{CH}_{3} \mathrm{CN}\right)_{2} \mathrm{Cl}_{2}$, in THF, toluene or DMF, and with $\mathrm{CuI}$ or $\mathrm{CuCN}$ additives (Scheme 5). Furthermore, tributylstannylalkyne 16 and vinyliodide $\mathbf{1 7 b}$ or $\mathbf{1 7} \mathbf{c}^{10}$ were coupled using $\left[\mathrm{PdCl}\left(\mathrm{PPh}_{3}\right)_{2} \mathrm{Bn}(15\right.$ $\mathrm{mol} \%$ ) and $\mathrm{CuI}(30 \mathrm{~mol} \%)$, in toluene at $85^{\circ} \mathrm{C}$, in a modest $30 \%$ yield to deliver enynes 19 or 20.11 These results showed that $(Z)$ or trisubstituted olefins 15, 17a, 17b and 17c did not undergo efficient Stille coupling reactions. 12
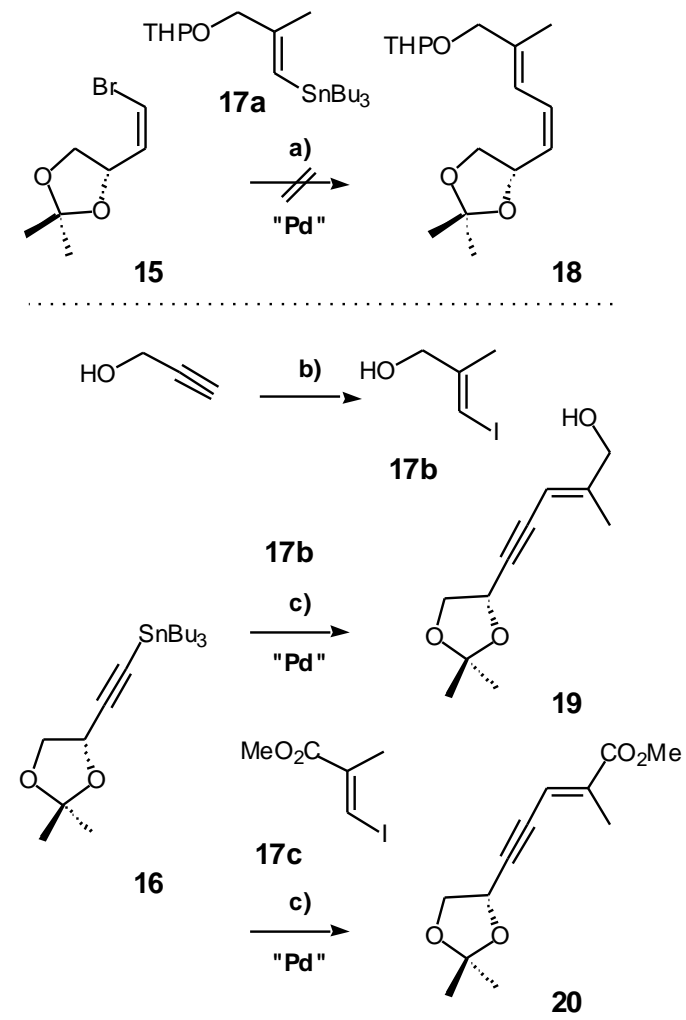

a) $\mathrm{Pd}\left(\mathrm{PPh}_{3}\right)_{4}$ or $\mathrm{Pd}\left(\mathrm{CH}_{3} \mathrm{CN}\right)_{2} \mathrm{Cl}_{2}(15 \mathrm{~mol} \%), \mathrm{CuI}(30 \mathrm{~mol} \%)$, THF, toluene or $\mathrm{DMF}, 85^{\circ} \mathrm{C}$. b) $\mathrm{Cp}_{2} \mathrm{ZrCl}_{2}$ (0.25 equiv.), $\mathrm{AlMe}_{3}$ (3 equiv.), $\mathrm{CH}_{2} \mathrm{Cl}_{2}, 20^{\circ} \mathrm{C}$ $24 \mathrm{~h}$, then $\mathrm{I}_{2}\left(1.2\right.$ equiv.), $0^{\circ} \mathrm{C}, \mathbf{2 5 \%}$. c) $\mathbf{1 7 b}$ or $\mathbf{1 7 c}, \mathrm{PdCl}\left(\mathrm{CH}_{3} \mathrm{CN}\right)_{2} \mathrm{Bn}(15$ mol\%), $\mathrm{CuI}(30 \mathrm{~mol} \%)$, toluene, $85^{\circ} \mathrm{C}, 12 \mathrm{~h}, \mathbf{3 0 \%}$.

\section{Scheme 5}

Consequently, we turned to the Shen method.13 Compound 14 was first transformed in 3 steps via alcohol 21 into the corresponding 1,1-dibromo-alkene derivative 22 in $45 \%$ overall yield (Scheme 6). 14 Compounds 22 and 17a under Shen's conditions $\left[\mathrm{Pd}\left(\mathrm{PPh}_{3}\right)_{4}\right.$ or $\mathrm{Pd}\left(\mathrm{CH}_{3} \mathrm{CN}\right)_{2} \mathrm{Cl}_{2} / \mathrm{CuI} /$ diisopropylethylamine, in toluene or DMF at $85^{\circ} \mathrm{C}$ ] conducted to the expected enyne 23 in $63 \%$ yield. 15 


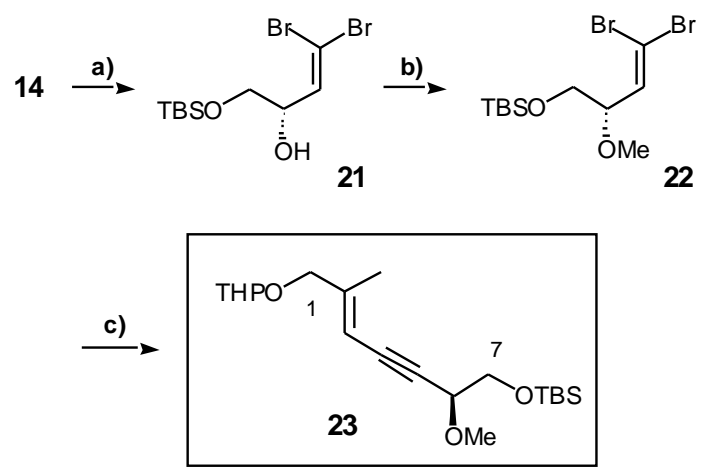

a) i- Amberlyst $15, \mathrm{MeOH}, 20^{\circ} \mathrm{C}, 12 \mathrm{~h}, \mathbf{7 1 \%}$. ii- TBSCl, $\mathrm{Im}, \mathrm{CH}_{2} \mathrm{Cl}_{2} 20^{\circ} \mathrm{C}$, $10 \mathrm{~h}, \mathbf{9 1 \%}$. b) MeI (excess), THF/DMF 8:1, NaH (1.3 equiv.), $0^{\circ} \mathrm{C}, 3 \mathrm{~h}$, 70\%. c) 17a, $\mathrm{Pd}\left(\mathrm{CH}_{3} \mathrm{CN}\right)_{2} \mathrm{Cl}_{2}(15 \mathrm{~mol} \%), \mathrm{CuI}(30 \mathrm{~mol} \%)$, DIPEA (1.5 equiv.), DMF, $85^{\circ} \mathrm{C}, 12 \mathrm{~h}, \mathbf{6 3 \%}$.

\section{Scheme 6}

In summary, preparation of the Eastern part 23, precursor of the dienyl fragment 4 of herbimycin A 1, was achieved in 19\% overall yield for 5 steps from the known glyceraldehyde derivative $\mathbf{1 3}$.

The elaboration of Southern C8-C15 sub-unit 5 started from the commercially available hydroxy-ester $\mathbf{2 4}$; classical transformations gave access, via ester 25, to the $\alpha, \beta$-unsaturated aldehyde 26 in $50 \%$ overall yield for 6 steps (Scheme 7).

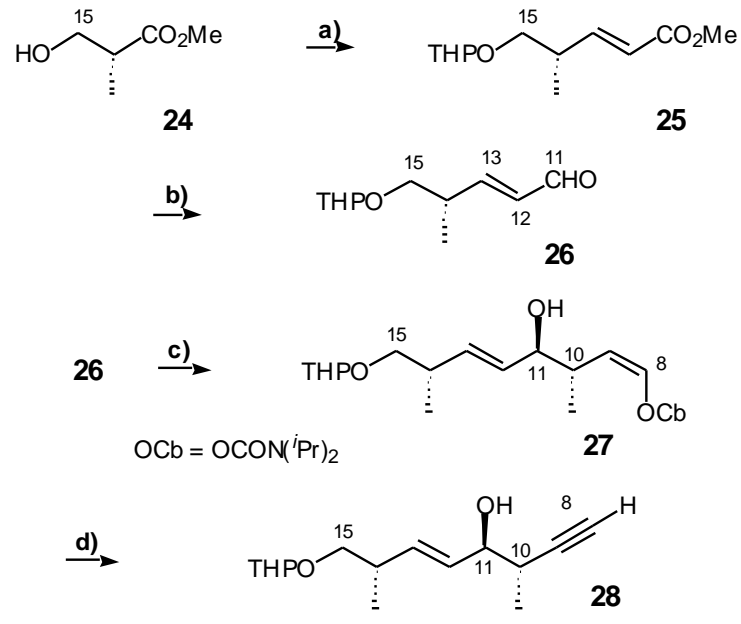

a) i- DHP, APTS, $20^{\circ}, 12 \mathrm{~h}, \mathbf{9 5 \%}$. ii- LAH $(0.8$ equiv. $) / \mathrm{THF}, 0^{\circ} \mathrm{C}, 3 \mathrm{~h}$, 85\%. iii- IBX (2.2 equiv.), DMSO, $20^{\circ} \mathrm{C}, 2 \mathrm{~h}$, quantitative. iv$\left(\mathrm{C}_{6} \mathrm{H}_{5}\right)_{3} \mathrm{P}=\mathrm{CHCO}_{2} \mathrm{Me} /$ toluene, $50^{\circ} \mathrm{C}, 10 \mathrm{~h}, \mathbf{8 1 \%}$. b) i- DIBAL-H $(2.1$ equiv.)/THF, $-78^{\circ} \mathrm{C}, 1 \mathrm{~h}, \mathbf{8 0} \%$. ii- IBX (2.2 equiv.), DMSO, $20^{\circ} \mathrm{C} 2 \mathrm{~h}, \mathbf{9 5 \%}$. c) $(E)$-crotyl (diisopropyl)carbamate (2.6 equiv.), $1.6 \mathrm{M} n$-BuLi in hexane (2.4 equiv.), (-)-sparteine (2.3 equiv.), $-78^{\circ} \mathrm{C} 10 \mathrm{~min}$, then $-78^{\circ} \mathrm{C} 3 \mathrm{~h}$ for crystallisation. Then $\mathrm{Ti}(\mathrm{O} i \mathrm{Pr})_{4}(6$ equiv. $)$, pentane, $-50^{\circ} \mathrm{C}$ and $-78^{\circ} \mathrm{C}, 30 \mathrm{~min}$ for transmetallation, $26-78^{\circ} \mathrm{C}, 2 \mathrm{~h}, \mathbf{8 5 \%}$. d) tert-BuLi (3.1 equiv.)/Et $2 \mathrm{O}$, $78^{\circ} \mathrm{C}, 45 \mathrm{~min}, \mathbf{7 0 \%}$.

\section{Scheme 7}

Elaboration of the C15-C8 skeleton was initiated by an enantioselective Hoppe crotylation 16 of aldehyde 26 to afford the pure 
vinyl carbamate 27 in $85 \%$ yield (100\%de) (Scheme 7). Further tertBuLi (3 equiv.) treatment of $\mathbf{2 7}$ then delivered the expected acetylenic derivative $\mathbf{2 8}$ in $70 \%$ yield.

Since it results from a Hoppe aldehyde allylation reaction, compound 28 exhibits an anti relationship between the methyl group at $\mathrm{C} 10$ and the $\mathrm{OH}$ function at $\mathrm{C} 11$; therefore, an inversion of the $\mathrm{C} 11$ centre was envisaged under the Mitsunobu conditions.

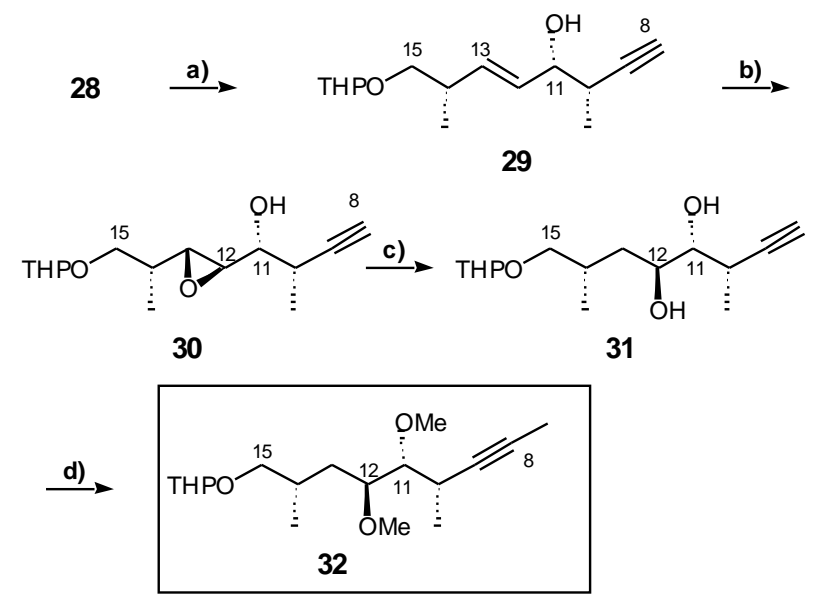

a)- i- DIAD (1.2 equiv.), $\mathrm{PPh}_{3}$ (1.2 equiv.), $\mathrm{PhCO}_{2} \mathrm{H}$ (1.2 equiv.) toluene, $78^{\circ} \mathrm{C}, 10 \mathrm{~min}, \mathbf{4 0 \%}$. ii- $\mathrm{LiAlH}_{4} \mathrm{THF}, 20^{\circ} \mathrm{C} \mathbf{8 0} \%$. b) D-(-)-diethyltartratre (1.2 equiv.), $\mathrm{Ti}(\mathrm{O} i \mathrm{Pr})_{4}$ (1.2 equiv.), TBHP (2 equiv.), $\mathrm{CH}_{2} \mathrm{Cl}_{2},-25^{\circ} \mathrm{C} 15 \mathrm{~h}$, 85\%. c) DIBAL-H (2.1 equiv.), THF, $0^{\circ} \mathrm{C} 5$ h, 60\%. d) $n$-BuLi (3.2 equiv.), MeI excess, THF, $-78^{\circ} \mathrm{C}->20^{\circ} \mathrm{C} 12 \mathrm{~h}, \mathbf{7 0 \%}$.

\section{Scheme 8}

The C11 hydroxyl function is both in an allylic and a homopropargylic position; as a consequence, application of the Mitsunobu reaction to 28 conducted to the inverted benzoyl ester in a fair $40 \%$ yield when the temperature was kept at $-78^{\circ} \mathrm{C}$ for $10 \mathrm{~min}$. Generation of alcohol 29 was then obtained by $\mathrm{LiAlH}_{4}$ reduction ( $80 \%$ yield, Scheme 8 ).

At this stage, installation of the secondary $\mathrm{C} 12$ alcohol function was envisaged in a two steps sequence. Stereoselective matched Sharpless epoxidation 17 of 29 using D-(-)-diethyltartarate gave epoxide $\mathbf{3 0}$ in $85 \%$ yield as a pure isomer (the corresponding diastereomer was not observed). Reduction of $\mathbf{3 0}$ under DIBAL-H/THF conditions 18 the furnished the anti-1,2-diol 31 in $60 \%$ yield (the corresponding anti1,3-diol was isolated in $9 \%$ yield).

Subsequent methylation of $\mathbf{3 1}$ was then carried out using 3.2 equivalents of $n$-BuLi and an excess of MeI to furnish dimethoxy derivative 32 in $70 \%$ yield. 19

In this sequence, and in spite of a modest yield for the Mitsunobu reaction, preparation of Southern fragment 32 was achieved in 10\% overall yield for 11 steps from the commercially available ester $\mathbf{2 4 . 2 0}$ Structural proof for $\mathbf{3 1}$ was obtained by NMR analysis of lactol 33, prepared in a three-step sequence (Scheme 9). 


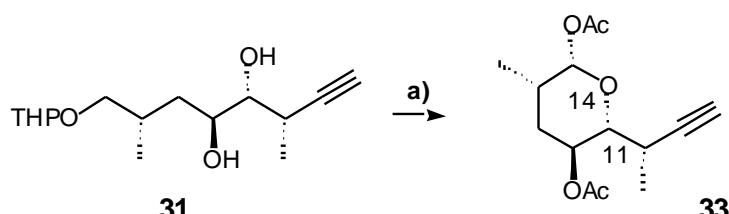

a) i- Amberlyst $15, \mathrm{MeOH} 25^{\circ} \mathrm{C} 15 \mathrm{~h}$. ii- IBX (2.2 equiv.), DMSO $0^{\circ} \mathrm{C} 1.5$ h. iii- $\mathrm{Ac}_{2} \mathrm{O}$, pyridine, $\mathbf{6 3 \%}$ yield for 3 steps.

\section{Scheme 9}

In conclusion, preparation of Northern fragment $\mathbf{2}$ of herbimycin A $\mathbf{1}$ was achieved in 55\% yield for 3 steps. Synthesis of the Eastern part 23, precursor of dienyl fragment 4, was performed in $19 \%$ overall yield for 5 steps from the known glyceraldehyde derivative 13 . Finally, elaboration of the $\mathrm{C} 8 \mathrm{-C} 15$ chain 32, precursor of aldehyde 3, was accomplished in $3.4 \%$ overall yield for 13 steps.

The final synthesis of the Hsp90 inhibitor, herbimycin A 1, is under study in our laboratory.

Acknowledgements: The CNRS, UCP and ESCOM are acknowledged for financial support. We gratefully thank Pfizer Laboratories for a doctoral fellowship to S. C.-A.

\section{References and Notes:}

1) Zhang, H.; Burrows, F. J. Mol. Med. 2004, 82, 488. Wegele, H.; Müller, L.; Buchner, J. Rev. Physiol. Biochem. Pharmacol. 2004, 151, 1. Jones, D. T.; Addison, E.; North, J. M.; Lowdell, M. W.; Hoffbrand, A. V.; Mehta, A. B.; Ganeshaguru, K.; Folarin, N. I.; Wickremasinghe, R. G. Blood 2004, 103, 1855. Bagatell, R.; Whitesell, L. Mol. Cancer Ther. 2004, 3, 1021. Bedin, M.; Gaben, A.-M.; Saucier, C.; Mester, J. Int. J. Cancer 2004, 109, 643.

2) Omura, S.; Iwai, Y.; Takahashi, Y.; Sadakane, A.; Nakagawa, A.; Oiwa, H.; Hasegawa, Y.; Ikai, T. J. Antibiotics 1979, 32, 255. Omura, S.; Nakagawa, A.; Sadakane, N. Tetrahedron Lett. 1979, 4323. Furusaki, A.; Matsumoto, T.; Nakagawa, A.; Omura, S. J. Antibiotics 1980, 33, 781. Iwai, Y.; Nakagawa, A.; Sadakane, N.; Omura, S.; Oiwa, H.; Matsumoto, S.; Takahashi, M.; Ikai, T.; Ochiai Y. J. Antibiotics 1980, 33, 1114. Shibata, 
K.; Satsumabayashi, S.; Nakagawa, A.; Omura, S. J. Antibiotics 1986, 39, 1630.

3) Nakata, M.; Osumi, T.; Ueno, A.; Kimura, T.; Tamai, T.; Tatsuta, K. Bull. Chem. Soc. Jpn 1992, 65, 2974. Nakata, M.; Osumi, T.; Ueno, A.; Kimura, T.; Tamai, T.; Tatsuta, K. Tetrahedron Lett. 1991, 32, 6015. Carter, K. D.; Panek, J. S. Org. Lett. 2004, 6, 55. Synthesis of advanced fragments: Eshelman, J. E.; Epps, J. L.; Kallmerten, J. Tetrahedron Lett. 1993, 34, 749. Martin, S. F.; Dodge, J. A.; Burgess, L. E.; Limberakis, C.; Hartmann, M. Tetrahedron, 1996, 52, 3229. Martin, S. F.; Limberakis, C.; Burgess, L. E.; Hartmann, M. Tetrahedron, 1996, 52, 3229.

4) 2: ${ }^{1} \mathrm{H} \mathrm{NMR}\left(270 \mathrm{MHz}, \mathrm{CDCl}_{3}\right) \delta 7.60(\mathrm{~d}, J=2.5 \mathrm{~Hz}, 1 \mathrm{H}), 7.50$ $(\mathrm{d}, J=2.5 \mathrm{~Hz}, 1 \mathrm{H}), 3.85\left(\mathrm{~s}, 3 \mathrm{H}, \mathrm{CH}_{3}, \mathrm{OMe}\right), 3.60$ (s, 3H, $\mathrm{CH}_{3}$, OMe). MS (GC, EI), m/z $309\left(\mathrm{M}^{+}\right)$.

5) Jackson, D. Y. Synth. Commun. 1988, 18, 337.

6) Corey, E.-J.; Fuchs, P. L. Tetrahedron Lett. 1972, 36, 3769.

7) Jiang, B.; Ma P. Synth. Commun. 1995, 25, 3641. Jackson, D. Y. Synth. Commun. 1988, 18, 337.

8) Uenishi, J.-i.; Kawahama, R.; Shiga, Y.; Yonemitsu, O.; Tsuji, J. Tetrahedron Lett. 1996, 37, 6759.

9) Booker-Milburn, K. I.; Heffernan, G. D.; Parsons, P. J. J. Chem. Soc., Chem. Commun. 1992, 350. Barbero, A.; Cuadrado, P.; Fleming, I.; Gonzalez, A. M.; Pulido, F. J. J. Chem. Soc., Chem. Commun. 1992, 351.

10) Baker, R.; Castro, J. L. J. Chem. Soc., Perkin Trans I 1990, 47.

11) 19: $1 \mathrm{H} \mathrm{NMR}\left(270 \mathrm{MHz}, \mathrm{CDCl}_{3}\right) \delta 5.54$ (s wide, $\left.1 \mathrm{H}\right), 4.82(\mathrm{dd}, J$ $=6.4,6.2 \mathrm{~Hz}, 1 \mathrm{H}), 4.11(\mathrm{dd}, J=7.8,6.2 \mathrm{~Hz}, 1 \mathrm{H}), 4.04(\mathrm{~s}, 2 \mathrm{H})$, $3.86(\mathrm{dd}, J=7.8,6.4 \mathrm{~Hz}, 1 \mathrm{H}), 1.81\left(\mathrm{~s}, 3 \mathrm{H}, \mathrm{CH}_{3}\right), 1.57(\mathrm{~s}, 1 \mathrm{H}$, $\mathrm{OH}), 1.43$ (s, 3H, $\left.\mathrm{CH}_{3}\right), 1.33\left(\mathrm{~s}, 3 \mathrm{H}, \mathrm{CH}_{3}\right)$.

12) "The Stille reaction", Vittorio Farina, Venkat Krishnamurthy and William J. Scott, John Wiley \& Sons, INC, 1998.

13) Shen, W.; Wang, L. J. Org. Chem. 1999, 64, 8873.

14) 22: $1 \mathrm{H} \mathrm{NMR}\left(270 \mathrm{MHz}, \mathrm{CDCl}_{3}\right) \delta 6.29(\mathrm{~d}, J=8.0 \mathrm{~Hz}, 1 \mathrm{H})$, $3.93(\mathrm{~m}, 1 \mathrm{H}), 3.63(\mathrm{~m}, 2 \mathrm{H}), 3.29\left(\mathrm{~s}, 3 \mathrm{H}, \mathrm{CH}_{3}, \mathrm{OCH}_{3}\right), 0.82[\mathrm{~s}$, $\left.9 \mathrm{H}, 3 \mathrm{CH}_{3}, \mathrm{SiC}\left(\mathrm{CH}_{3}\right)_{3}\right], 0.01$ [s, $\left.6 \mathrm{H}, 2 \mathrm{CH}_{3}, \mathrm{Si}\left(\mathrm{CH}_{3}\right)_{2}\right]$. 13C NMR (67.8 MHz, $\left.\mathrm{CDCl}_{3}\right) \delta 137.2(\mathrm{CH}), 92.4(\mathrm{C}), 82.2$ $(\mathrm{CH}), 64.4\left(\mathrm{CH}_{2}\right), 57.2\left(\mathrm{CH}_{3}, \mathrm{OCH}_{3}\right), 25.8\left[3 \mathrm{CH}_{3}, \mathrm{SiC}(\underline{\mathrm{CH}} 3)_{3}\right]$, 
$18.3\left[\mathrm{C}, \mathrm{SiC}\left(\mathrm{CH}_{3}\right)_{3}\right],-4.0,-4.5\left[2 \mathrm{CH}_{3}, \mathrm{Si}\left(\mathrm{CH}_{3}\right)_{2}\right]$. MS (CI, NH $\left.{ }_{3}\right), m / z 392\left(\mathrm{MH}^{+}+\mathrm{NH}_{3}\right), 375\left(\mathrm{MH}^{+}\right)$.

15) 23: ${ }^{1} \mathrm{H}$ NMR $\left(400 \mathrm{MHz}, \mathrm{CDCl}_{3}\right) \delta 5.61$ (broad s, $\left.1 \mathrm{H}\right), 4.61(\mathrm{t}, J$ $=3.3 \mathrm{~Hz}, 1 \mathrm{H}), 4.19(\mathrm{~d}, J=14.4 \mathrm{~Hz}, 1 \mathrm{H}), 4.17(\mathrm{~m}, 1 \mathrm{H}), 3.94(\mathrm{~d}$, $J=14.4 \mathrm{~Hz}, 1 \mathrm{H}), 3.84(\mathrm{~m}, 1 \mathrm{H}), 3.79(\mathrm{dd}, J=10.5,6.5 \mathrm{~Hz}, 1 \mathrm{H})$, $3.77(\mathrm{dd}, J=10.5,5.5 \mathrm{~Hz}, 1 \mathrm{H}), 3.51(\mathrm{~m}, 1 \mathrm{H}), 3.45\left(\mathrm{~s}, 3 \mathrm{H}, \mathrm{CH}_{3}\right.$, $\left.\mathrm{OCH}_{3}\right), 1.89\left(\mathrm{~s}, 3 \mathrm{H}, \mathrm{CH}_{3}\right), 1.73-1.58\left(\mathrm{~m}, 6 \mathrm{H}, 3 \mathrm{CH}_{2}\right), 0.82[\mathrm{~s}$, $\left.9 \mathrm{H}, 3 \mathrm{CH}_{3}, \mathrm{SiC}\left(\mathrm{CH}_{3}\right)_{3}\right], 0.01$ [s, $\left.6 \mathrm{H}, 2 \mathrm{CH}_{3}, \mathrm{Si}\left(\mathrm{CH}_{3}\right)_{2}\right]$.

${ }^{13 \mathrm{C}} \mathrm{NMR}\left(67.8 \mathrm{MHz}, \mathrm{CDCl}_{3}\right) \delta 148.5(\mathrm{C}), 105.5(\mathrm{CH}), 98.1$ $(\mathrm{CH}), 90.0(\mathrm{C}), 84.2(\mathrm{C}), 73.9(\mathrm{CH}), 70.6\left(\mathrm{CH}_{2}\right), 66.7\left(\mathrm{CH}_{2}\right)$, $62.5\left(\mathrm{CH}_{2}\right), 57.3\left(\mathrm{CH}_{3}, \mathrm{OCH}_{3}\right), 30.9\left(\mathrm{CH}_{2}\right), 26.4\left[3 \mathrm{CH}_{3}\right.$, $\left.\mathrm{SiC}\left(\mathrm{CH}_{3}\right)_{3}\right], 25.8\left(\mathrm{CH}_{2}\right), 19.7\left(\mathrm{CH}_{2}\right), 18.9\left[\mathrm{C}, \mathrm{SiC}\left(\mathrm{CH}_{3}\right)_{3}\right],-4.8$ $-4-7\left[2 \mathrm{CH}_{3}, \mathrm{Si}\left(\mathrm{CH}_{3}\right)_{2}\right]$

MS (CI, NH 3$), m / z 386\left(\mathrm{MH}^{+}+\mathrm{NH}_{3}\right), 369\left(\mathrm{MH}^{+}\right)$.

IR $\left(\mathrm{CCl}_{4}\right)$ 2929, 2856, 2360, 2341, 1578, 1463, 1129, $869 \mathrm{~cm}^{-1}$. Anal. Calcd for $\mathrm{C}_{20} \mathrm{H}_{36} \mathrm{O}_{4} \mathrm{Si}, 368.58$, C $65.17 \%$, H 9.84\%. Found C 65.35\%, H, 9.97\%.

16) Hoppe, D. Angew. Chem. Int. Ed. 1984, 23, 932. Hoppe, D.; Zschage, O. Angew. Chem. Int. Ed. 1989, 28, 69. Zschage, O.; Hoppe, D. Tetrahedron 1992, 48, 5657. Hoppe, D.; Hense, T. Angew. Chem. Int. Ed. 1997, 36, 2282.

17) Katsuki, T.; Sharpless, K. B. Tetrahedron Lett. 1979, 20, 4733. Sharpless, K. B.; Verhoeven, T. R. Aldrichimica Acta 1979, 12, 63. Katsuki, T.; Sharpless, K. B. J. Am. Chem. Soc. 1980, 102, 5974.

18) Hansen, R. M. Chem. Rev. 1991, 91, 437. Dai, L.; Lou, B.; Zhang, Y.; Guo, G. Tetrahedron Lett. 1986, 4343. Sharpless, B. M.; Caron, M. J. Org. Chem. 1985, 50, 1557.

19) 32: $1 \mathbf{H}$ NMR $\left(270 \mathrm{MHz}, \mathrm{CDCl}_{3}\right) \delta$ (two diastereomers) 4.55 (m, 1H), $3.80(\mathrm{~m}, 1 \mathrm{H}), 3.65(\mathrm{~m}, 1 \mathrm{H}), 3.50(\mathrm{~m}, 1 \mathrm{H}), 3.39,3.37$ (2s, $\left.3 \mathrm{H}, \mathrm{CH}_{3}, \mathrm{CH}_{3} \mathrm{O}\right), 3.28,3.26\left(2 \mathrm{~s}, 3 \mathrm{H}, \mathrm{CH}_{3}, \mathrm{CH}_{3} \mathrm{O}\right), 3.20(\mathrm{~m}$, $1 \mathrm{H}), 3.03(\mathrm{~m}, 1 \mathrm{H}), 3.24(\mathrm{~m}, 1 \mathrm{H}), 2.79(\mathrm{~m}, 1 \mathrm{H}), 2.03(\mathrm{~m}, 1 \mathrm{H})$, $1.78\left(\mathrm{~s}, 3 \mathrm{H}, \mathrm{CH}_{3}\right), 1.73-1.58\left(\mathrm{~m}, 6 \mathrm{H}, 3 \mathrm{CH}_{2}\right), 1.68(\mathrm{~m}, 1 \mathrm{H}), 1.42$, (m, $1 \mathrm{H}), 1.18\left(\mathrm{~d}, J=7.0 \mathrm{~Hz}, 3 \mathrm{H}, \mathrm{CH}_{3}\right), 0.99(\mathrm{~d}, J=6.9 \mathrm{~Hz}, 3 \mathrm{H}$, $\mathrm{CH}_{3}$ ). ${ }^{13} \mathbf{C}$ NMR $\left(67.5 \mathrm{MHz}, \mathrm{CDCl}_{3}\right) \delta$ (two diastereomers) 98.7, $98.5(\mathrm{CH}), 81.4(\mathrm{CH}), 80.5(\mathrm{CH}), 80.1(\mathrm{CH}), 79.0(\mathrm{C})$, 72.4, $72.3\left(\mathrm{CH}_{2}\right), 62.4(\mathrm{C}), 61.5,61.2\left(\mathrm{CH}_{3}, \mathrm{CH}_{3} \mathrm{O}\right), 57.9,57.8$ $\left(\mathrm{CH}_{3}, \mathrm{CH}_{3} \mathrm{O}\right), 33.1\left(\mathrm{CH}_{2}\right), 30.4,30.2\left(\mathrm{CH}_{2}\right), 29.2(\mathrm{CH}), 28.8$ 
(CH), 25.4, $25.3\left(\mathrm{CH}_{2}\right), 19.3,19.2\left(\mathrm{CH}_{2}\right), 18.3\left(\mathrm{CH}_{3}\right), 17.8$ $\left(\mathrm{CH}_{3}\right), 3.5\left(\mathrm{CH}_{3}\right)$. Anal. Calcd for $\mathrm{C}_{18} \mathrm{H}_{32} \mathrm{O}_{4}, 312.44 ; \mathrm{C}, 69.19$; $\mathrm{H}, 10.32$. Found $\mathrm{C}, 69.03 ; \mathrm{H}, 10.56$.

20) A second approach was also explored from the same starting material 24. This route used more conventional transformation to reach the desired fragment in a $6 \%$ overall yield for 17 steps, with a Sharpless dihydroxylation and a Brown crotylation to introduce the stereogenic centres.

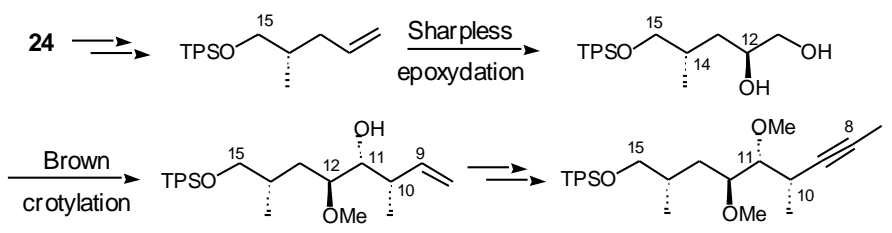

